\title{
BMJ Open Screening for chronic kidney disease in a community-based diabetes cohort in rural Guatemala: a cross-sectional study
}

\author{
David Flood, ${ }^{1,2}$ Pablo Garcia, ${ }^{1}$ Kate Douglas, ${ }^{1}$ Jessica Hawkins, ${ }^{1}$ Peter Rohloff ${ }^{1,3}$
}

To cite: Flood D, Garcia P, Douglas $\mathrm{K}$, et al. Screening for chronic kidney disease in a community-based diabetes cohort in rural Guatemala: a 2018;8:e019778. doi:10.1136/ bmjopen-2017-019778

- Prepublication history for this paper is available online. To view these files, please visit the journal online (http://dx.doi org/10.1136/bmjopen-2017019778).

Received 25 September 2017 Revised 3 November 2017 Accepted 24 November 2017 cross-sectional study. BMJ Open

\section{ABSTRACT}

Objective Screening is a key strategy to address the rising burden of chronic kidney disease (CKD) in lowincome and middle-income countries. However, there are few reports regarding the implementation of screening programmes in resource-limited settings. The objectives of this study are to (1) to share programmatic experiences implementing CKD screening in a rural, resource-limited setting and (2) to assess the burden of renal disease in a community-based diabetes programme in rural Guatemala. Design Cross-sectional assessment of glomerular filtration rate (GFR) and urine albumin.

Setting Central Highlands of Guatemala.

Participants We enrolled 144 adults with type 2 diabetes in a community-based CKD screening activity carried out by the sponsoring institution.

Outcome measures Prevalence of renal disease and risk of CKD progression using Kidney Disease: Improving Global Outcomes definitions and classifications.

Results We found that $57 \%$ of the sample met GFR and/ or albuminuria criteria suggestive of CKD. Over half of the sample had moderate or greater increased risk for CKD progression, including nearly $20 \%$ who were classified as high or very high risk. Hypertension was common in the sample (42\%), and glycaemic control was suboptimal (mean haemoglobin A1c $9.4 \% \pm 2.5 \%$ at programme enrolment and $8.6 \% \pm 2.3 \%$ at time of CKD screening). Conclusions The high burden of renal disease in our patient sample suggests an imperative to better understand the burden and risk factors of CKD in Guatemala. The implementation details we share reveal the tension between evidence-based CKD screening versus screening that can feasibly be delivered in resource-limited global settings.

\section{INTRODUCTION}

'Wuqu' Kawoq, Santiago Sacatepéquez, Sacatepéquez, Guatemala

${ }^{2}$ Department of Medicine, University of Minnesota, Minneapolis, MN, USA

${ }^{3}$ Division of Global Health Equity, Brigham and Women's Hospital, Boston, MA, USA

Correspondence to Dr Peter Rohloff; peter@wuqukawoq.org
Chronic kidney disease (CKD) is a critical global health problem. ${ }^{1-3}$ The worldwide CKD prevalence rate is $11 \%-13 \%{ }^{4}$ From 2005 to 2015 , deaths due to CKD rose from 0.9 to 1.2 million per year, primarily owing to increases in CKD caused by diabetes and hypertension. ${ }^{5}$ Data on CKD are limited in low-income and middle-income countries (LMICs), but age-adjusted prevalence and mortality rates may be greater than in high-income countries. ${ }^{67}$ The causes of CKD in

\section{Strengths and limitations of this study}

This study is one of the first to describe the implementation of a chronic kidney disease (CKD) screening programme in a rural area of a lowincome or middle-income country.

- The primary strength of this study relates to the practical barriers that were overcome to implement a guideline-directed CKD screening programme in this setting.

- We investigated CKD in a small diabetes sample of 144 people, limiting the generalisability of our results.

- Our results could have overestimated the overall prevalence of CKD among people with diabetes in rural Guatemala, given that we sampled from a single institution's diabetes cohort rather than using a population-based sampling strategy.

- Our sample was predominantly composed of women, which reflects known challenges in enrolling men in chronic disease programmes in Latin America.

LMICs are heterogeneous and incompletely understood, and most individuals are undiagnosed. ${ }^{289}$ A growing proportion of those with CKD in LMICs develop end-stage renal disease (ESRD), yet most do not have access to life-saving renal replacement therapy (RRT). ${ }^{1011}$

The region of interest in this study is Latin America. Here, marked disparities exist with regard to the nephrology workforce and RRT rates. ${ }^{12}$ Latin America has the highest CKD death rate in the world, ${ }^{5}$ and diabetes is the leading cause of ESRD. ${ }^{12}$ Recent high-quality evidence from Mexico suggests that diabetes is a potent risk factor for CKD and death from renal disease in this region. ${ }^{13}$

\section{CKD screening and management in resource- limited settings}

Scaling up screening is an important strategy to address the burden of CKD in LMICs. ${ }^{14-16}$ International clinical guidelines recommend CKD screening for individuals with risk factors such as diabetes, using laboratory 
assessments of glomerular filtration rate (GFR) and urine albumin excretion. ${ }^{17-20}$ In the case of diabetes, interventions shown to slow disease progression for individuals who screen positive for CKD include glycaemic control, blood pressure management and renoprotection with ACE inhibitors or angiotensin receptor blockers. ${ }^{21}$

However, there are many barriers to implementing CKD screening in resource-limited settings. Screening for CKD may be cost-effective in high-income countries in high-risk patients such as those with diabetes,${ }^{22}$ but the cost-effectiveness in LMICs is uncertain. This is in large part because international CKD screening guidelines require access to specialised laboratory testing, ${ }^{21}$ which is frequently unavailable at the primary care level in LMICs. ${ }^{23}$ Furthermore, many national health systems in LMICs are not equipped or funded to deliver integrated care for individuals with CKD once they are detected by screening. ${ }^{23}{ }^{24}$ Finally, there are few published reports documenting the practical details of implementing CKD screening programmes in LMICs. Scaling up CKD care requires that all implementers more readily share their experiences in designing and evaluating CKD screening programmes.

\section{Study objectives}

This study describes the implementation and outcomes of a small, community-based CKD screening programme for patients with type 2 diabetes in rural Guatemala. The objectives are (1) to share our programmatic experiences implementing CKD screening in a rural, resource-limited setting and (2) to assess the burden of renal disease in a community-based diabetes programme in rural Guatemala.

\section{METHODS}

\section{Setting}

This study was carried out in Guatemala by the non-governmental healthcare organisation Wuqu' Kawoq (www. wuqukawoq.org). Since 2007, the organisation has operated a free, comprehensive type 2 diabetes programme serving approximately 250 rural patients in the Central Highlands of Guatemala. We previously have published implementation details and a clinical protocol of this programme. ${ }^{25}$ The programme is community based with the majority of patients entering on a first-come, firstserved basis based on programme capacity.

Guatemala is a Latin American country with a population of approximately 16 million. Approximately $40 \%$ of Guatemalans are indigenous Maya, ${ }^{26}$ a predominantly rural ethnolinguistic group with poverty rates approaching $80 \%$ or greater, ${ }^{27}$ poor general health indicators ${ }^{28}$ and limited access to healthcare. ${ }^{29}{ }^{30}$ The rural health system in Guatemala is fragmented and highly privatised, and people with chronic diseases face profound barriers when seeking healthcare. ${ }^{31}$ Individuals with diabetes typically have experienced years of uncontrolled disease prior to presentation to our institution's clinic. ${ }^{32}$
While the epidemiology and risk factors for CKD have not been well studied in Guatemala, crude renal failure mortality rates are among the highest in the Americas. ${ }^{33}$ In addition to rising rates of traditional CKD risk factors like diabetes, some studies show that the newly described disease entity 'CKD of non-traditional causes' may also be affecting rural Guatemalan populations. ${ }^{34}{ }^{35}$ Guatemala also has one of the world's highest rates of child malnutrition, ${ }^{36}$ which is a risk factor for reduced renal function. ${ }^{37}$ With 54 total nephrologists, Guatemala has only 3.3 nephrologists per million population, below the Latin America average. ${ }^{12}$ Nevertheless, the number of people on RRT has increased rapidly, and Guatemala now has one of the highest per capita peritoneal dialysis rates in the world. ${ }^{38}$

\section{Implementation of a community-based CKD screening and management programme in Guatemala}

Our organisation previously had screened pragmatically for CKD in patients with diabetes using measurement of serum creatinine and manual assessment of urine dipstick protein. ${ }^{18}{ }^{39}$ In 2014, after an increase in morbidity and mortality secondary to diabetes nephropathy including more than a dozen cases of ESRD, we redesigned our screening protocol to include assessment of both serum creatinine and urine albumin/creatinine excretion as recommended in the Kidney Disease: Improving Global Outcomes (KDIGO) guidelines. ${ }^{21}$

Implementing a KDIGO-aligned CKD screening programme posed significant logistical and financial challenges in our rural Guatemalan context. Reliable assays were only available at urban laboratory facilities, so we funded individual patient transportation or paid for couriers to transport specimens collected in rural villages. Assay costs were approximately US $\$ 8$ per test for urine creatinine and urine albumin, and US $\$ 2.50$ per test for serum creatinine. Collection and transportation fees approximately doubled these per-assay costs. These expenses were significant given that our overall budget for comprehensive diabetes care is approximately US $\$ 250$ per patient per year, a sum we finance through donor fundraising.

Patients identified with CKD, including moderately increased albuminuria (albumin-to-creatinine ratio (ACR) of $30-300 \mathrm{mg} / \mathrm{g}$ ), received guideline-directed medical care to prevent CKD progression, ${ }^{21}$ including management of hyperglycaemia with oral antidiabetic medications and insulin, treatment of albuminuria with ACE inhibitors, efforts to control hypertension with ACE inhibitors and other classes of antihypertensive agents, and integration of a behavioural intervention to improve exercise and diet. As we have described elsewhere,${ }^{25}$ in our clinical protocols, we target haemoglobin A1c (HbA1c) $<8.0 \%$ and blood pressure $<140 / 90 \mathrm{~mm} \mathrm{Hg}$; these targets are less strict than those recommended in some guidelines but rational given our rural setting with minimal access to emergency services in the event of medication-related adverse effects. In line with KDIGO guidance, ${ }^{21}$ we 
monitored creatinine and potassium after initiating or escalating ACE inhibitor therapy, which added further costs to the programme. Of note, angiotensin receptor blockers are cost-prohibitive in our setting.

We referred patients with GFR below $30 \mathrm{~mL} /$ $\min / 1.73 \mathrm{~m}^{2}$ and who wished to be considered for dialysis to the National Center for Chronic Renal Disease, a public health facility in Guatemala City that provides nephrology care, including haemodialysis and continuous ambulatory peritoneal dialysis (CAPD). Our organisation provides patient navigation and primary care services for rural patients with diabetes who carry out home-based CAPD. $^{40}$

\section{Study design and sample}

We used data collected from a retrospective chart review of CKD screening among individuals with diabetes treated at our institution to carry out a cross-sectional assessment of GFR and urine albumin. Inclusion criteria were age $\geq 18$ years, enrolment in our diabetes programme, and at least one measurement of HbAlc between 3 January 2014 (when the CKD screening programme was implemented) and 5 January 2015 (when data extraction began). Exclusion criteria were incomplete albuminuria data, diagnosis of type 1 diabetes, diagnosis of gestational diabetes or current use of dialysis. A total of 157 patients met the study's inclusion criteria. Thirteen patients were subsequently excluded: two patients with type 1 diabetes, one patient with gestational diabetes, three patients receiving dialysis (one of whom also had type 1 diabetes) and eight patients with missing urine albumin data. A final list of 144 patients was identified that comprise the sample in this paper.

\section{Laboratory assessment of kidney function}

At contracting commercial laboratories, serum creatinine was assessed with the Microlab 300 analyser (ELITech, Paris, France). Urine ACR was carried out using the Cobas 6000/c501 analyser (Roche, Basel, Switzerland). Specimens were collected, packaged and shipped to in accordance with the guidelines of Mayo Medical Laboratories. ${ }^{41}$

\section{Data analysis and definitions}

Participant data were extracted from our electronic medical record to a spreadsheet and then imported to Stata V.13 for statistical analyses. Two independent data coders reviewed the spreadsheet for errors. We first calculated baseline characteristics using descriptive statistics. We compared the underlying characteristics of patients in the sample versus those with missing urine albumin data using the two-sample rank-sum test and Fisher's exact test.

Second, we assessed CKD staging using GFR and albuminuria categories as recommended by KDIGO. ${ }^{21}$ We calculated GFR using the 2009 CKD-Epidemiology Collaboration (CKD-EPI) equation. ${ }^{42}$ Due to inherent limitations of our organisation's screening programme, ACR and serum creatinine testing often did not occur on the same day. Mapping of risk stratification of disease progression was performed using KDIGO nomenclature and summarised visually with a waffle chart. Finally, we evaluated the use and indication of ACE inhibitors between albuminuria category and hypertension diagnosis. KDIGO has released detailed guidelines on the management of hypertension and albuminuria in people with diabetes, ${ }^{21} 43$ including a weaker recommendation for treatment of moderately increased albuminuria versus severely increased albuminuria. For the purposes of this analysis, we assumed that an ACE inhibitor was indicated if a patient had a diagnosis of hypertension and/or ACR greater than $30 \mathrm{mg} / \mathrm{g}$.

\section{Consent}

A waiver of consent was obtained as the research presented no more than minimal risk of harm to subjects and involved no procedures for which written consent is normally required outside of the research context.

\section{RESULTS}

\section{Patient characteristics}

Baseline demographic and clinical information is presented in table 1 . The patient sample was predominantly women (83\%), mostly Mayan speaking $(61 \%)$ and had low levels of educational attainment (median 2 years). At the date of creatinine measurement, mean age was $54.6 \pm 11.8$ years, and patients reported living with diabetes for a median of 6 (IQR 3-10) years. Median enrolment in the clinical diabetes programme was 1.5 (IQR 0.2-2.8) years, and mean HbA1c at enrolment was $9.4 \% \pm 2.5 \%$. At most recent HbAlc measurement, the mean was $8.6 \% \pm 2.3 \%$, and $47 \%$ of patients had met the predefined programmatic target of $\leq 8.0 \%$. Forty-two per cent of the sample carried a diagnosis of hypertension, and $67 \%$ of patients had blood pressure $<140 / 90 \mathrm{~mm} \mathrm{Hg}$ at the most recent reading. A substantial proportion of patients had body mass index in the overweight $(72 \%)$ and obese range (29\%) range. Regarding medications, $90 \%$ were prescribed metformin, 53\% a sulfonylurea, 22\% insulin, $33 \%$ an ACE inhibitor and $15 \%$ at least one additional antihypertensive agent that was not an ACE inhibitor. In comparing characteristics of the sample to the eight patients excluded due to missing urine ACR, there were no significant differences in most variables (age, gender, initial HbA1c, most recent HbA1c, diagnosis of hypertension), but GFR was lower among those with missing albuminuria values.

\section{CKD indicators}

Risk of CKD progression according to the KDIGO system in our sample of patients with diabetes is summarised in figure 1. A majority $(77.1 \%)$ of the sample had at least mildly decreased GFR $\left(<90 \mathrm{~mL} / \mathrm{min} / 1.73 \mathrm{~m}^{2}\right)$, and $33.4 \%$ had GFR $<60 \mathrm{~mL} / \mathrm{min} / 1.73 \mathrm{~m}^{2}$ (G3a or worse). Most $(65.2 \%)$ of the sample had ACR values in the normal range of $<30 \mathrm{mg} / \mathrm{g}$. Moderately increased albuminuria 
Table 1 Demographic and clinical profile

\begin{tabular}{|c|c|}
\hline Characteristic $(n=144)$ & Value* \\
\hline Age (years) & $54.6 \pm 11.8$ \\
\hline Female (\%) & 83 \\
\hline \multicolumn{2}{|l|}{ Language preference $(n=134)$} \\
\hline Mayan Kaqchikel or K'iche' (\%) & 61 \\
\hline Spanish (\%) & 39 \\
\hline Years of schooling (years, $n=132$ ) & $2(0-4)$ \\
\hline Years with diabetes (years, $n=139$ ) & $6(3-10)$ \\
\hline Years in clinical diabetes programme & $1.5(0.2-2.8)$ \\
\hline \multicolumn{2}{|l|}{$\mathrm{HbA1c}$} \\
\hline At enrolment (\%) & $9.4 \pm 2.5$ \\
\hline Current (\%) & $8.6 \pm 2.3$ \\
\hline$\leq 8.0 \%(\%)$ & 47 \\
\hline \multicolumn{2}{|l|}{ Blood pressure } \\
\hline Diagnosis of hypertension (\%) & 42 \\
\hline Systolic blood pressure (mm Hg) & $128 \pm 21$ \\
\hline Diastolic blood pressure $(\mathrm{mm} \mathrm{Hg})$ & $77 \pm 11$ \\
\hline$<140 / 90 \mathrm{~mm} \mathrm{Hg}(\%)$ & $67 \%$ \\
\hline \multicolumn{2}{|l|}{ Body mass index $(n=132)$} \\
\hline Mean $\left(\mathrm{kg} / \mathrm{m}^{2}\right)$ & $27.6 \pm 4.7$ \\
\hline$\geq 25(\%)$ & 72 \\
\hline$\geq 30(\%)$ & 29 \\
\hline \multicolumn{2}{|l|}{ Medication prescriptions } \\
\hline Metformin (\%) & 90 \\
\hline Sulfonylurea (\%) & 53 \\
\hline Insulin (\%) & 22 \\
\hline ACE inhibitors (\%) & 33 \\
\hline $\begin{array}{l}\text { Other antihypertensive agent (beta- } \\
\text { blocker, calcium-channel blocker or } \\
\text { thiazide diuretic) }\end{array}$ & $15 \%$ \\
\hline
\end{tabular}

*Normally distributed values are described as mean \pm SD and nonnormally distributed values as median (IQR).

$\mathrm{HbA1c}$, haemoglobin A1c.

$(30-300 \mathrm{mg} / \mathrm{g})$ was observed in $26.4 \%$ and severely increased albuminuria $(>300 \mathrm{mg} / \mathrm{g})$ in $8.4 \%$ of patients. Overall, $57 \%$ had GFR $<60 \mathrm{~mL} / \mathrm{min} / 1.73 \mathrm{~m}^{2}$ or ACR of $>30 \mathrm{mg} / \mathrm{g}$.

Using the KDIGO classification for risk of CKD progression, $43.1 \%$ of patients were classified as low risk, $38.9 \%$ as moderately increased risk, $9.0 \%$ as high risk and $9.0 \%$ as very high risk. The median time interval between ACR and creatinine screening assessments was 56 (IQR 17-115) days.

\section{Use of ACE inhibitors}

Table 2 displays the proportion of patients prescribed an ACE inhibitor by albuminuria category and hypertension diagnosis prior to implementation of the KDIGO-aligned CKD screening programme. Sixty-six per cent $(40 / 61)$ of patients with hypertension had an ACE inhibitor prescription. Among those patients with a diagnosis of hypertension,
$53 \%(8 / 15)$ of participants with moderately increased microalbuminuria and $100 \%(9 / 9)$ with severely increased microalbuminuria had been prescribed an ACE inhibitor at the time of albuminuria assessment. Among patients who did not have a diagnosis of hypertension, ACE inhibitors had been prescribed in $17 \%(4 / 23)$ of cases with moderately increased microalbuminuria and $0 \%(0 / 3)$ of cases with severely increased microalbuminuria. There were three patients in the sample who had been prescribed an ACE inhibitor but who did not have hypertension or elevated albuminuria.

\section{DISCUSSION}

This was a retrospective chart review of a community-based CKD screening programme for adults with type 2 diabetes in rural Guatemala. Our programme's implementation details and results raise key implications for CKD screening efforts in rural, resource-limited global settings.

First, we report a very high burden of renal disease among our sample of adults with type 2 diabetes in rural Guatemala. Nearly $20 \%$ of individuals were classified as high or very high risk for CKD progression, and $57 \%$ of the sample met GFR and/or albuminuria criteria suggestive of CKD. For comparison, the prevalence of CKD among people with diabetes in the USA is approximately $26 \%,{ }^{44}$ and in high-risk cohorts in other LMICs has ranged from $19 \%$ to $49 \% .^{9}$ Surprisingly, most participants with reduced GFR had normoalbuminuria $(65.2 \%)$, a finding at odds with the prevalence of normoalbuminuric CKD described in high-quality longitudinal and cross-sectional studies. ${ }^{45}$ Additionally, the proportion of participants with hypertension $(42 \%)$ was lower than in other cohorts of people with type 2 diabetes. ${ }^{46}$ We are uncertain if these findings reflect a unique feature of the diabetes epidemic among Maya indigenous populations or are an artefact of sampling and data collection. In other settings, emerging evidence suggests that the profile of people with CKD differs across LMICs. ${ }^{47}$ Given the limited data on the epidemiology of CKD in Guatemala, ${ }^{12} 3548$ our findings show that it is imperative to better study the burden and risks factors of CKD in this setting through representative population-based surveys.

Second, while our study was not designed or powered to analyse associations of CKD with glycaemic control or blood pressure, it was notable that the mean HbAlc was quite high $(9.4 \% \pm 2.5 \%$ at programme enrolment and $8.6 \% \pm 2.3 \%$ at time of CKD screening) and that a sizeable proportion of the sample (42\%) had a diagnosis of hypertension. However, one-third of those with hypertension had not been prescribed an ACE inhibitor, one-third of the sample did not meet blood pressure goal of $<140 / 90 \mathrm{~mm}$ $\mathrm{Hg}$, and only a small number of patients ( $15 \%$ of sample) were taking a separate antihypertensive agent. These findings reiterate the fundamental importance of quality management of diabetes and hypertension in addressing CKD in our context and other resource-limited settings.

Finally, we describe the practical barriers in implementing our CKD screening programme, including cost, 


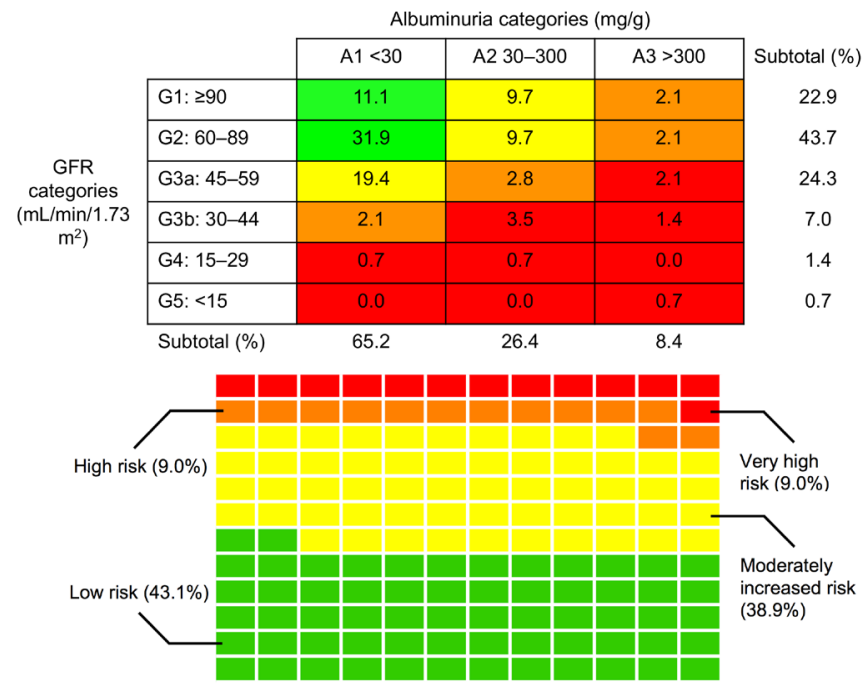

Figure 1 Risk of chronic kidney disease (CKD) progression. (Top panel) Risk map for CKD progression. Cells coded by Kidney Disease: Improving Global Outcomes (KDIGO) risk level as follows: green, low risk; yellow, moderately increased risk; orange, high risk; red, very high risk. Glomerular filtration rate (GFR) was calculated using CKD-Epidemiology Collaboration equation. KDIGO designations are as follows: A1, normal to mildly increased albuminuria; A2, moderately increased albuminuria; A3, severely increased albuminuria; G1, normal or high GFR; G2, mildly decreased GFR; G3a, mildly to moderately decreased; G3b, moderately to severely decreased GFR; G4, severely decreased GFR; G5, kidney failure. (Bottom panel) Waffle chart of KDIGO CKD risk progression categories. Each rectangle denotes a single patient in the sample $(n=144)$. Cells are coded by KDIGO risk as in the top panel.

geographical constraints and lack of access to laboratory facilities. Three additional implementation issues that arose as we designed and implemented our screening programme are worth describing in more detail: (1) use of urine dipsticks, (2) point-of-care (POC) creatinine testing and (3) monitoring adverse effects of ACE inhibitor treatment. Regarding urine dipsticks, some clinical groups suggest using dipstick proteinuria as a CKD screening test in resource-limited settings. ${ }^{18}{ }^{39}$ However, dipsticks are insensitive to low levels of urine albumin ${ }^{49}$ and perform poorly when interpreted visually, ${ }^{50}$ as typically must be done in rural clinics. Low-cost dipsticks to detect albuminuria are in development, ${ }^{5253}$ but currently available models are insensitive and require use of an analyser. ${ }^{54}$ Regarding POC serum creatinine assessment, our institution has had success using POC tests for $\mathrm{HbAlc}^{25}$ and reliable POC serum creatinine

Table 2 Proportion of sample prescribed ACE inhibitor by albuminuria category and hypertension diagnosis

\begin{tabular}{lll}
\hline $\begin{array}{l}\text { Albuminuria categories } \\
\text { (mg/g) }\end{array}$ & $\begin{array}{l}\text { Patients without Patients with } \\
\text { hypertension }\end{array}$ & hypertension \\
\hline$<30(\mathrm{~A} 1)$ & $5 \%(3 / 57)$ & $62 \%(23 / 37)^{\star}$ \\
\hline $30-300$ (A2) & $17 \%(4 / 23)^{\star}$ & $53 \%(8 / 15)^{\star}$ \\
$>300$ (A3) & $0 \%(0 / 3)^{\star}$ & $100 \%(9 / 9)^{\star}$ \\
\hline Total & $8 \%(7 / 83)$ & $66 \%(40 / 61)$ \\
\hline
\end{tabular}

Kidney Disease: Improving Global Outcomes designations are as follows: A1, normal to mildly increased albuminuria; A2, moderately increased albuminuria; $A 3$, severely increased albuminuria.

${ }^{*} \mathrm{ACE}$ inhibitor was assumed to be indicated for the treatment of hypertension and/or elevated microalbuminuria. assays are available. ${ }^{55}$ However, cost and durability represent major limitations to the widespread implementation of POC serum creatinine assays in global CKD screening programmes. Regarding ACE inhibitors, reports from LMICs often do not describe protocols, training or costs associated with monitoring adverse effects of these drugs.

These implementation and screening challenges reveal the tension between evidence-based CKD screening and screening that can feasibly be delivered in rural, under-resourced areas. There is a dearth of guidelines specifically tailored for CKD screening in resource-limited settings. Important exceptions include the International Diabetes Federation, ${ }^{18}$ a CKD chapter in Partners In Health's field manual for non-communicable diseases in in Rwanda ${ }^{39}$ and CKD screening reports from a handful of other LMICs. ${ }^{56-58}$ We urge other global health workers to share their experiences implementing CKD screening programmes and adapting international CKD guidelines to local contexts.

In our clinical programming, our pragmatic response to the work described in this paper has been to prioritise hypertension and diabetes control as the most practical CKD response in our context. We have de-emphasised the more costly screening with ACR and reinvested in quality improvement initiatives relating to management of hypertension and diabetes. This view of addressing CKD by prioritising treatment of comorbid non-communicable diseases echoes similar proposals from WHO. ${ }^{59}$ We continue to contemplate the role of low-cost urine dipstick for field screening of proteinuria, while acknowledging that a CKD screening approach without ACR will fail to detect normotensive patients with diabetes with moderately increased 
albuminuria who would benefit from ACE inhibitors (13\% of our sample). ${ }^{60}$ Given the extent of CKD in our sample, we recognise that aggressive treatment of hypertension with ACE inhibitors entails a high risk of side effects. To minimise harm, we have decided to aggressively monitor side effects in line with KDIGO standards, ${ }^{21}$ though this practice involves increased costs.

Our research has several weaknesses and limitations. We investigated CKD in a small community-based diabetes cohort rather than a large, representative sample, so our results cannot be generalised within Guatemala or to other countries. Due to challenges assessing CKD, other reports from LMICs also have used convenience sampling. ${ }^{957}$ Compared with the overall rural diabetes population in Guatemala, our reported CKD prevalence could have been overestimated, as our programme occasionally accepts referral patients with known diabetes complications. At the same time, CKD prevalence could have been underestimated as we excluded patients with ESRD from our analysis. Our sample was predominantly composed of women (83\%), reflecting known difficulties enrolling men in chronic disease programmes in Latin America. ${ }^{61}$

A final set of weaknesses relate to our laboratory assessment of CKD. Due to the programmatic, retrospective nature of this research, we did not often measure serum creatinine and urine ACR on the same date. Like other groups ${ }^{957}$ we calculated CKD prevalence rates and the risk of progression based on a single, cross-sectional assessment; not conducting confirmatory testing after 3 months, as indicated in KDIGO guidelines, ${ }^{21}$ may have overestimated the true CKD prevalence rate and risk profile of the sample. ${ }^{62}$ Additionally, our contracting commercial laboratory could not confirm the specific assay used to assess serum creatinine or traceability to IDMS. Finally, we used the 2009 CKD-EPI equation to calculate GFR, but this equation has not been validated in Guatemala. Our decision to use this equation is supported by KDIGO guidelines, ${ }^{21}$ which recommend using CKD-EPI unless an alternative estimation has been found to be superior for a specific target population.

Acknowledgements We thank Sandy Mux, Carol Teleguario, Waleska Lopez, German Obispo and Luisa Ixjotop for assisting in study activities and for delivering high-quality diabetes care in rural Guatemala.

Contributors DF and PR designed the study, secured funding and carried out fieldwork. DF, PG, KD and JH extracted and cleaned the data. DF, PG and PR analysed the data. DF and PG wrote the initial draft with input from PR. All authors were involved in the manuscript revisions and approved the final version.

Funding This work was supported from operating funds of Wuqu' Kawoq and a grant from the Center for Primary Care at Harvard Medical School.

Competing interests None declared.

Patient consent Not required.

Ethics approval This study was approved by the Institutional Review Board of Wuqu' Kawoq/Maya Health Alliance (WK-2017-002) and conforms to the principles embodied in the Declaration of Helsinki.

Provenance and peer review Not commissioned; externally peer reviewed.

Data sharing statement De-identified replication data are available through Wuqu' Kawoq's Dataverse site at doi:10.7910/DVN/NSE8BB.

Open Access This is an Open Access article distributed in accordance with the Creative Commons Attribution Non Commercial (CC BY-NC 4.0) license, which permits others to distribute, remix, adapt, build upon this work non-commercially, and license their derivative works on different terms, provided the original work is properly cited and the use is non-commercial. See: http://creativecommons.org/ licenses/by-nc/4.0/

(C) Article author(s) (or their employer(s) unless otherwise stated in the text of the article) 2018. All rights reserved. No commercial use is permitted unless otherwise expressly granted.

\section{REFERENCES}

1. Jha V, Arici M, Collins AJ, et al. Conference Participants. Understanding kidney care needs and implementation strategies in low- and middle-income countries: conclusions from a "Kidney Disease: Improving Global Outcomes" (KDIGO) Controversies Conference. Kidney Int 2016;90:1164-74.

2. Stanifer JW, Muiru A, Jafar TH, et al. Chronic kidney disease in low- and middle-income countries. Nephrol Dial Transplant 2016;31:868-74.

3. Neuen BL, Chadban SJ, Demaio AR, et al. Chronic kidney disease and the global NCDs agenda. BMJ Glob Health 2017;2:e000380.

4. Hill NR, Fatoba ST, Oke JL, et al. Global prevalence of chronic kidney disease - a systematic review and meta-analysis. PLoS One 2016;11:e0158765.

5. GBD 2015 Mortality and Causes of Death Collaborators. Global, regional, and national life expectancy, all-cause mortality, and causespecific mortality for 249 causes of death, 1980-2015: a systematic analysis for the global burden of disease study 2015. Lancet 2016;388:1459-544.

6. Mills KT, Xu Y, Zhang W, et al. A systematic analysis of worldwide population-based data on the global burden of chronic kidney disease in 2010. Kidney Int 2015;88:950-7.

7. Institute for Health Metrics and Evaluation (IHME). GBD Compare data visualization. Seattle, WA: IHME, University of Washington, 2016. (accessed 15 Jul 2017).

8. Jha V, Garcia-Garcia G, Iseki K, et al. Chronic kidney disease: global dimension and perspectives. Lancet 2013;382:260-72.

9. Ene-lordache B, Perico N, Bikbov B, et al. Chronic kidney disease and cardiovascular risk in six regions of the world (ISN-KDDC): a cross-sectional study. Lancet Glob Health 2016;4:e307-e319.

10. Liyanage T, Ninomiya T, Jha V, et al. Worldwide access to treatment for end-stage kidney disease: a systematic review. Lancet 2015;385:1975-82

11. Ashuntantang G, Osafo C, Olowu WA, et al. Outcomes in adults and children with end-stage kidney disease requiring dialysis in sub-Saharan Africa: a systematic review. Lancet Glob Health 2017;5:e408-e417.

12. Cusumano AM, Rosa-Diez GJ, Gonzalez-Bedat MC. Latin American dialysis and transplant registry: experience and contributions to end-stage renal disease epidemiology. World $J$ Nephrol 2016;5:389-97.

13. Alegre-Díaz J, Herrington W, López-Cervantes M, et al. Diabetes and cause-specific mortality in Mexico city. N Engl J Med 2016;375:1961-71

14. Perico N, Bravo RF, De Leon FR, et al. Screening for chronic kidney disease in emerging countries: feasibility and hurdles. Nephrol Dial Transplant 2009;24:1355-8.

15. George C, Mogueo A, Okpechi I, et al. Chronic kidney disease in low-income to middle-income countries: the case for increased screening. BMJ Glob Health 2017;2:e000256.

16. Cusumano AM, González Bedat MC. Chronic kidney disease in Latin America: time to improve screening and detection. Clin J Am Soc Nephrol 2008;3:594-600.

17. American Diabetes Association. Standards of medical care in diabetes-2017: summary of revisions. Diabetes Care 2017;40(Suppl 1):S1-135.

18. International Diabetes Federation. Global guideline for type 2 diabetes. Brussels, Belgium: International Diabetes Federation, 2012.

19. Inker LA, Astor BC, Fox CH, et al. KDOQI US commentary on the $2012 \mathrm{KDIGO}$ clinical practice guideline for the evaluation and management of CKD. Am J Kidney Dis 2014;63:713-35.

20. Asociación Latinoamericana de Diabetes. Guías ALAD sobre el diagnóstico, control y tratamiento de la diabetes mellitus tipo 2 con medicina basada en evidencia edición 2013. Revista de la ALAD 2013:1-142.

21. Kidney Disease: Improving Global Outcomes. KDIGO 2012 clinical practice guideline for the evaluation andmanagement of chronic kidney disease. Kidney Int 2013;3:i-150. 
22. Komenda P, Ferguson TW, Macdonald K, et al. Cost-effectiveness of primary screening for CKD: a systematic review. Am J Kidney Dis 2014;63:789-97.

23. Bello AK, Levin A, Tonelli M, et al. Assessment of global kidney health care status. JAMA 2017;317:1864-81.

24. Samb B, Desai N, Nishtar S, et al. Prevention and management of chronic disease: a litmus test for health-systems strengthening in low-income and middle-income countries. Lancet 2010;376:1785-97.

25. Flood D, Mux S, Martinez B, et al. Implementation and outcomes of a comprehensive type 2 diabetes program in rural Guatemala. PLOS One 2016;11:e0161152.

26. Instituto Nacional de Estadística. Encuesta nacional de condiciones de vida 2014: tomo I. Guatemala, Central America: Instituto Nacional de Estadística, 2016

27. Sanchez SM, Scott K, Humberto Lopez J. Guatemala - closing gaps to generate more inclusive growth: systematic country diagnostic. Washington, DC: The World Bank, 2015.

28. PAHO. Health in the Americas, 2012. Guatemala: PAHO, 2012. Country Volume.

29. Cerón A, Ruano AL, Sánchez S, et al. Abuse and discrimination towards indigenous people in public health care facilities: experiences from rural Guatemala. Int $J$ Equity Health 2016;15:77

30. Hautecoeur M, Zunzunegui MV, Vissandjee B. [Barriers to accessing health care services for the indigenous population in Rabinal, Guatemala]. Salud Publica Mex 2007;49:86-93.

31. Chary A, Rohloff P, eds. Privatization and the new medical pluralism: shifting healthcare landscapes in Maya Guatemala. Lanham, Maryland: Lexington Press, 2015.

32. Chary A, Greiner M, Bowers C, et al. Determining adult type 2 diabetes-related health care needs in an indigenous population from rural Guatemala: a mixed-methods preliminary study. BMC Health Serv Res 2012;12:476.

33. PAHO and WHO. Renal failure and chronic kidney disease (CKD) mortality visualization. 2014 http://www.paho.org/hq/index.php? option=com_content\&view=article\&id=9402\&ltemid=41166\&lang=en (accessed 4 Feb 2017).

34. Laux TS, Barnoya J, Cipriano E, et al. Prevalence of chronic kidney disease of non-traditional causes in patients on hemodialysis in southwest Guatemala. Rev Panam Salud Publica 2016;39:186-93.

35. Laux TS, Barnoya J, Guerrero DR, et al. Dialysis enrollment patterns in Guatemala: evidence of the chronic kidney disease of non-traditional causes epidemic in Mesoamerica. BMC Nephrol 2015;16:54.

36. Black RE, Victora CG, Walker SP, et al. Maternal and child undernutrition and overweight in low-income and middle-income countries. Lancet 2013;382:427-51.

37. Luyckx VA, Brenner BM. Birth weight, malnutrition and kidneyassociated outcomes--a global concern. Nat Rev Nephrol 2015:11:135-49.

38. Jain AK, Blake P, Cordy P, et al. Global trends in rates of peritoneal dialysis. J Am Soc Nephrol 2012;23:533-44.

39. Partners In Health. "The PIH guide to chronic care integration for endemic non-communicable diseases: Rwanda edition": Partners in Health, 2011.

40. Flood DC, Chary AN, Austad K, et al. A patient navigation system to minimize barriers for peritoneal dialysis in rural, low-resource settings: case study from Guatemala. Kidney Int Rep 2017;2:762-5.

41. Mayo Medical Laboratories, Mayo Clinic. Collect, package, \& ship. 2017 http://www.mayomedicallaboratories.com/specimen/ (accessed 24 Mar 2017).

42. Levey AS, Stevens LA, Schmid $\mathrm{CH}$, et al. A new equation to estimate glomerular filtration rate. Ann Intern Med 2009;150:604-12.

43. Kidney Disease: Improving Global Outcomes. KDIGO clinical practice guideline for the management of blood pressure in chronic kidney disease. Kidney Int Supp/ 2012;2.
44. Afkarian $M$, Zelnick $L R$, Hall $Y N$, et al. Clinical manifestations of kidney disease among us adults with diabetes, 1988-2014. JAMA 2016;316:602-10.

45. Pugliese G, Solini A, Bonora E, et al. chronic kidney disease in type 2 diabetes: lessons from the renal insufficiency and cardiovascular events (RIACE) Italian multicentre study. Nutr Metab Cardiovasc Dis 2014:24:815-22.

46. Colosia AD, Palencia R, Khan S. Prevalence of hypertension and obesity in patients with type 2 diabetes mellitus in observational studies: a systematic literature review. Diabetes Metab Syndr Obes 2013;6:327-38

47. Anand S, Zheng Y, Montez-Rath ME, et al. Do attributes of persons with chronic kidney disease differ in low-income and middleincome countries compared with high-income countries? Evidence from population-based data in six countries. BMJ Glob Health 2017;2:e000453.

48. Unidad Nacional de Atención al Enfermo Renal Crónico (UNAERC). Pacientes activos por programa y consulta del mes según bioestadísticas. 2015 http://unaerc.gob.gt/estadisticas/pacientesactivos-por-programa-y-consulta-del-mes-segun-bioestadisticas/ (accessed 24 Oct 2017).

49. Park JI, Baek H, Kim BR, et al. Comparison of urine dipstick and albumin:creatinine ratio for chronic kidney disease screening: a population-based study. PLoS One 2017;12:e0171106.

50. Rumley A. Urine dipstick testing: comparison of results obtained by visual reading and with the Bayer CLINITEK 50. Ann Clin Biochem 2000;37(Pt 2):220-1.

51. Waugh JJ, Bell SC, Kilby MD, et al. Optimal bedside urinalysis for the detection of proteinuria in hypertensive pregnancy: a study of diagnostic accuracy. BJOG 2005;112:412-7.

52. Tugirimana PL, Delanghe JR. Development of an affordable dyestained microalbuminuria screening test. Nephrol Dial Transplant 2009;24:1485-90.

53. Grand Challenges Canada. Urine dipsticks as a screening tool for chronic renal disease (CRD). http://www.grandchallenges.ca/ grantee-stars/0518-01-10/ (accessed 28 Jul 2017).

54. McTaggart MP, Newall RG, Hirst JA, et al. Diagnostic accuracy of point-of-care tests for detecting albuminuria: a systematic review and meta-analysis. Ann Intern Med 2014;160:550-7.

55. Gbinigie O, Price CP, Heneghan $\mathrm{C}$, et al. Creatinine point-of-care testing for detection and monitoring of chronic kidney disease: primary care diagnostic technology update. Br J Gen Pract 2015;65:608-9.

56. Sharma SK, Ghimire A, Carminati S, et al. Management of chronic kidney disease and its risk factors in eastern Nepal. Lancet Glob Health 2014;2:e506-e507.

57. Cravedi P, Sharma SK, Bravo RF, et al. Preventing renal and cardiovascular risk by renal function assessment: insights from a cross-sectional study in low-income countries and the USA. BMJ Open 2012;2:bmjopen-2012-001357.

58. Sumaili EK, Cohen EP. Screening for chronic kidney disease in subSaharan Africa. Lancet 2010;376:418.

59. WHO. Action plan for the prevention and control of non-communicable diseases, 2013-2020. Geneva, Switzerland: World Health Organization, 2013.

60. Ravid M, Lang R, Rachmani R, et al. Long-term renoprotective effect of angiotensin-converting enzyme inhibition in non-insulindependent diabetes mellitus. A 7-year follow-up study. Arch Intern Med 1996;156:286-9.

61. Fort MP, Castro M, Peña L, et al. Opportunities for involving men and families in chronic disease management: a qualitative study from Chiapas, Mexico. BMC Public Health 2015;15:1019.

62. Bottomley MJ, Kalachik A, Mevada C, et al. Single estimated glomerular filtration rate and albuminuria measurement substantially overestimates prevalence of chronic kidney disease. Nephron Clin Pract 2011;117:348-52. 